\title{
Erratum
}

\section{Erratum for Lun et al., Spatially Heterogeneous Choroid Plexus Transcriptomes Encode Positional Identity and Contribute to Regional CSF Production}

In the article "Spatially Heterogeneous Choroid Plexus Transcriptomes Encode Positional Identity and Contribute to Regional CSF Production” by Melody P. Lun, Matthew B. Johnson, Kevin G. Broadbelt, Momoko Watanabe, Young-jin Kang, Kevin F. Chau, Mark W. Springel, Alexandra Malesz, André M. M. Sousa, Mihovil Pletikos, Tai Adelita, Monica L. Calicchio, Yong Zhang, Michael J. Holtzman, Hart G. W. Lidov, Nenad Sestan, Hanno Steen, Edwin S. Monuki, and Maria K. Lehtinen, which appeared on pages 4903-4916 of the March 25, 2015 issue, the eleventh author first name was misspelled. The corrected author line is: Melody P. Lun, Matthew B. Johnson, Kevin G. Broadbelt, Momoko Watanabe, Young-jin Kang, Kevin F. Chau, Mark W. Springel, Alexandra Malesz, André M. M. Sousa, Mihovil Pletikos, Tais Adelita, Monica L. Calicchio, Yong Zhang, Michael J. Holtzman, Hart G. W. Lidov, Nenad Sestan, Hanno Steen, Edwin S. Monuki, and Maria K. Lehtinen. This has been corrected on the online PDF version.

DOI:10.1523/JNEUROSCI.1672-15.2015 